\title{
Towards a conflict prevention strategy applicable for real-time railway traffic management
}

\author{
Sofie Van Thielen ${ }^{1}$, Francesco Corman ${ }^{2}$, and Pieter Vansteenwegen ${ }^{* 1}$ \\ ${ }^{1}$ KU Leuven, Leuven Mobility Research Centre - CIB, Celestijnenlaan 300, 3001 Leuven \\ ${ }^{2}$ ETH Zurich, Institute for Transport Planning and Systems, Stefano Franscini Platz 5, Zurich \\ 8093, Switzerland
}

July 8, 2019

\begin{abstract}
In real-time, dispatchers have the challenging task to perform railway traffic management. However, it is almost impossible to anticipate the impact of their actions on the entire network. In order to support dispatchers in making decisions, a Traffic Management System (TMS) is required capable of predicting train movements, detecting conflicts and deciding on how to prevent these conflicts.

This paper presents a Conflict Prevention Strategy (CPS) that can easily be integrated into an industrial TMS. We base on previous research on CPS which included a retiming/reordering heuristic and a local rerouting optimization, and a 'dynamicimpact-zone' heuristic to determine impact of conflicts. This paper proposes a series of algorithmic improvements to design a new strategy, called iCPS, usable in practice and able to solve very large and complex networks, very quickly, and with good performance. Moreover, the degree of realism of the instances can be increased, including multiple business rules related to passenger delays, and cancelling trains in case of very large delays. This iCPS is tested on a large study area, and validated on an even larger network. The proposed iCPS outperforms the FCFS strategy by, on average, $51 \%$ in train delays and $22 \%$ in passenger delays while delivering solutions in 2.4 seconds on average and in 34.1 seconds at maximum. It also outperforms the previous CPS by up to $11 \%$ in solution quality.
\end{abstract}

Keywords: Conflict prevention; Dispatching; Traffic management system; Realtime scheduling

*pieter.vansteenwegen@kuleuven.be 


\section{Introduction}

The growing need for sustainable transport entails a growing interest in public transport. Therefore, the quality of train services should be enhanced such that railway companies attract more passengers. This requires a robust timetable and as few delays as possible in real-time. A timetable ensures that two trains should not conflict with one another, i.e. no two trains need the same part of the infrastructure at the same time. However, during daily operations, trains can suffer delays due to, e.g., overcrowded platforms. Once trains start deviating from their original schedule, conflicts can appear and should be prevented.

During the past years, many methods have been developed to improve real-time railway traffic management (e.g., Corman and Meng, 2013; Cacchiani et al., 2014; Pellegrini et al., 2016). Recently, some methods have been implemented in practice on limited networks (see e.g., Borndörfer et al., 2017). However, a large gap still exists between the state-ofthe-art traffic management in academic research and the state-of-the-art in practice. This illustrates that many challenges arise when putting this academic research in practice.

Some infrastructure managers use advanced Traffic Management Systems (TMS) as a decision support system for their dispatchers (Dolder et al., 2009). Such TMS is only capable of predicting train movements and detecting conflicts. There is no decision support available yet to prevent or resolve these conflicts. Therefore, a Conflict Prevention Module (CPM), which can be easily integrated into a TMS, is required. This module should include a Conflict Prevention Strategy (CPS) capable of resolving detected conflicts.

In previous work, we introduced a first version of an efficient CPS (Van Thielen et al., 2018). Our current paper proposes an improved CPS, capable of preventing, in a fast way, detected conflicts in large and complex networks. This CPS is closer to practice, making it suitable for online usage. The first contribution of this paper is that multiple business rules have been included under direct input of the industrial partner: the CPS is adapted to take into account the reuse of rolling stock. Secondly, several improvements and new parameters are introduced for speeding up the CPS calculations. Thirdly, the CPS is extended such that passenger numbers and/or canceling trains can be considered during optimization. Fourthly, in practice some parts of the network, such as large station areas integrating different stations, might need a tailored dispatching approach due to the specific structure. In this paper, we show our approach is still effective when such a large station area is considered as a black box where no interventions by our CPS are possible. The effectiveness of this improved and extended CPS is shown on a very large and complex network.

Section 2 starts by explaining some important definitions required for the remainder of the paper and includes a literature review. Section 3 explains the conflict resolution methodology and Section 4 discusses all improvements and extensions. Section 5 discusses the simulation framework and experimental setup and shows the experimental results applying the improved and extended strategy on a very large and complex network, and on an even larger network. The paper is concluded in Section 6 . 


\section{Definitions and literature review}

In this section, a number of concepts are discussed in order to define the scope of this research. A railway network is typically considered either on a microscopic or a macroscopic level. The microscopic level includes every detail concerning switches, tracks, signals, etc. This level is important for both train drivers and dispatchers. The macroscopic level is a simplification of the microscopic level and is often closely related to what passengers experience. In our research, a microscopic detail in infrastructure and timetable is used.

Every signal indicates the beginning or the end of a block section. The part of the infrastructure between two subsequent, similarly directed signals thus determines a block section. These two signals fix the size of the block section, which is typically around 1000 meters in Belgium. A switch enables a train to be guided from one track to another track.

The network can be further decomposed into two types of areas: a station area and a non-station area. A station area is located around a platform area where passengers can debark or embark a train. There are switch areas before and after the platforms such that many possibilities are available for a train when entering or departing the station area. A station area then typically consists of a switch area, a platform area and a switch area. This allows to reroute trains in station areas. All areas that are not station areas are assumed to be non-station areas.

In this research, trains are assumed to drive from signal to signal (and block section to block section). The signal gives information on the next sections. A green signal indicates that the next two sections are available. A double yellow signal implies that the following section is available, but the one following that section is occupied by another train. A red signal indicates that the next block section is occupied by another train. In practice a train has to slow down after a double yellow signal and stop at a red signal. In this research we will apply a common rule of thumb in planning and real-time railway management that trains can only move on if the two following block sections are available, i.e. a green signal.

According to Hansen and Pachl (2008), a block section is exclusively occupied by one train during a time interval, i.e. the blocking time theory. This time interval starts at the reservation time and ends at the release time. A conflict occurs whenever two blocks overlap in the time-distance diagram. The timetable determines the route of a train, i.e. the sequence of block sections. This research assumes that trains drive according to the blocking time theory.

This paper includes only a concise literature review. For a much more detailed discussion, we refer the interested reader to Van Thielen (2019). Recently, much research has been devoted to the Conflict Detection and Resolution problem (CDR). The CDR is the problem of resolving potential overlap of blocking times (within a time horizon of predicted future train operations) by retiming rescheduling or rerouting trains. The time horizon of train operations considered is typically ranging from 15 minutes up to an hour. The solutions are typically optimal, or near-optimal, with regard to some objective function. Despite the growing academic interest, only few approaches have been implemented in practice, implying that there is still a large gap between state-of-the-art in practice and in academics (Caimi et al., 2012), especially for dealing with large and complex networks. 
Macroscopic approaches have been developed, but these approaches do not deliver a clear and direct resolution to a particular conflict (Meng and Zhou, 2011). Therefore, a feasibility check needs to be included to assure that these resolutions can also be applied to the microscopic network. On the contrary, microscopic approaches deliver resolutions that are on the precision level required for dispatchers. These solutions can be immediately implemented in TMS.

There are several microscopic approaches in the literature to solve the CDR problem. The CDR problem can be modeled as a blocking (or no-store), no-wait job shop scheduling problem (Mascis and Pacciarelli, 2002). One research stream uses an alternative graph to model this problem (D'Ariano et al., 2007). In this stream, a state-of-the-art TMS has been developed, called ROMA, capable of rescheduling and iteratively rerouting trains in a limited network. Different approaches for rescheduling are included in this TMS (Corman et al., 2010, Corman et al., 2012a, Corman and D'Ariano, 2012b, Quaglietta et al., 2013, Samà et al., 2015).

Another stream of research solves the CDR problem by creating a mixed integer linear program (MILP) (Törnquist and Persson, 2007; Pellegrini et al., 2016). The decision support tool RECIFE uses RECIFE-MILP for solving the CDR problem (Pellegrini et al., 2016). This optimization model considers all possible routes for all trains, which is very time consuming. Therefore, Samà et al. (2017) introduce a metaheuristic algorithm to deal with the real-time train routing selection problem.

Some of the approaches have been targeting more or less directly the practical applicability in real life, by guaranteeing faster computation time (see for instance the combinatorial optimization improvements proposed in Fischetti and Monaci (2017)), and/or larger networks, tackled by decomposition (Corman et al. (2012a), Josyula et al. (2018), Lamorgese and Mannino (2015)). The largest instances considered in different papers range from multiple hours of prediction horizons (up to 12 in Lamorgese and Mannino (2015) ) or more than a hundred trains running.

Some approaches have been integrated in railway production systems. Montigel (2009) reports on the successful implementation of a small scale system, which improved the throughput of a bottleneck in the swiss railway network. A method based on the approaches presented in Lamorgese and Mannino (2015) and Lamorgese et al. (2016) is currently in operations in several systems in Italy, Latvia and Norway. Bettinelli et al. (2017) report on an industrial implementation of a greedy heuristic able to solve in real-time instances of up to 150 trains and two hours of prediction horizon. A general overview of those approaches is reported in Borndörfer et al. (2017), who summarize some successfully implemented approaches that are or have been in operations. An overview of the current academic and industrial challenges for railway traffic management is reported in Lamorgese et al. (2018). They specifically report on the need for industrial acceptance by including more and more precise business rules and processes; those are objectives of this paper.

The objective function considered in the CDR problem varies strongly. Mostly, research focuses on the total secondary or weighted train delays. Other objective functions found minimize the delay costs, the passenger annoyance or the passenger travel time (Törnquist and Persson, 2007; Cacchiani et al., 2014). This paper examines the differences between 
minimizing train delays, which is often desired by the infrastructure managers, and minimizing passenger delays, which is often wanted by the train operating companies and the passengers.

If a train has a large delay and still has many more conflicts in the near future, it can be considered to cancel it. Canceling (passenger) trains is often studied in case of disruptions, where one (or multiple) tracks are blocked for a time period (Louwerse and Huisman, 2014; Albrecht et al., 2013). To the best of our knowledge, canceling (passenger) trains has not been studied yet when only considering small disturbances in rail traffic, even when the delay would be larger to the planned headway of the train services.

The effectiveness on large and busy networks (more than multiple hundreds of trains per hour, for multiple hours) of the optimization approaches depends on a lot of industrial and computation requirements, as well as the need to be directly included into a control loop. To meet these requirements, also requested by Infrabel, the Belgian railway infrastructure manager, we proposed in a previous paper (Van Thielen et al., 2018) a CPS framework capable of delivering fast and good resolutions, when a conflict is detected by an advanced TMS. For every detected conflict, a dynamic impact zone is constructed and considered when evaluating the possible conflict resolutions. This dynamic impact zone is intended to include all trains and conflicts that are potentially affected by the resolution of the detected conflict. This is called a dynamic impact zone, because it will have a different size for each detected conflict and the size also depends on the time, location and traffic situation a conflict takes place.

Our current paper proposes several improvements and extensions such that this CPS framework and the concept of the dynamic impact zone can be applied in practice to even larger and more complex networks (which are still unmatched in the literature in terms of trains running per hours, at multiple hundreds). This requires algorithmic and implementation speedups, able to improve quality and speed for applicability in a practical setting. Furthermore, the CPS is shown able to deal with rolling stock connections and train cancellations. We also show that it is straightforward to use this approach with a different objective function, for instance minimizing passenger delays. The original goal of these modifications was to improve our CPS and make it usable in practice for large and complex networks, in fact the precise consideration of business objectives has been identified by Lamorgese et al., 2018 as a clear gap limiting the application of such systems. Nevertheless, these modifications also have scientific value. Any researcher wanting to monitor or evaluate efficiently the consequences of (the resolution of) a certain conflict, can benefit from our work. We indicate how to efficiently select the other conflicts (and trains) that should be considered and how these should be treated during the evaluation. Moreover, we show that it makes sense to use a distance limit when considering the consequences of a conflict. We also indicate the differences that can occur when considering passenger delays instead of train delays and discuss the benefits and drawbacks of canceling trains. 


\section{Conflict resolution methodology}

This section explains the basics of the Conflict Prevention Strategy (CPS) developed in Van Thielen et al. (2018).

When a conflict is detected by the TMS, it will be sent to the CPM. If the conflict is located in a station area, the rerouting optimization is started to try to minimize the train delays by giving alternative routes to (some of the) trains. As soon as an optimal solution is found, this is implemented. Otherwise, this optimization is limited by a maximum computation time. When this time limit is reached, the best found feasible solution is implemented; this is an improvement to Van Thielen et al. (2018) as we realised that even a suboptimal routing decision can deliver benefits with regards to the entire conflict prevention solution. If no feasible solution is found within the time limit, the current situation is given as input to the successive module, the retiming heuristic. The computation time spent in the rerouting optimization is included in the total computation time reported in Section 5, independent of if the optimization was successful or not. The complete mathematical model for the rerouting optimization is discussed in Section 4.1.

If the rerouting optimization does not resolve the conflict or if the conflict is not located in a station area, then a resolution based on retiming and/or reordering trains needs to be proposed. The retiming heuristic introduced in Van Thielen et al. (2018) is based on a dynamic impact zone, and therefore called the Dynamic Impact Zone (DIZ) heuristic. This dynamic impact zone is based: (1) on offline calculations determining which conflicts are most likely to take place and (2) on a set of potentially conflicting trains, which normally use the same infrastructure as the conflicting train within a time range of 20 minutes. Since the size of the impact zone (i.e. the number of conflicts/trains considered) is different for each detected conflict and the trains considered will also depend on their location at the time the conflict is expected, this impact zone is called dynamic. In other words, the amount of information considered dynamically depends on the characteristics of the network and timetable, learned by means of preliminary computations. Static approaches have the impact zone fixed in time, space, and amount of trains (for instance FCFS considers the conflict only).

The initial conflict is the conflict that is detected by TMS and sent to the CPM. A firstorder conflict is a conflict that contains (at least) one of the trains in the initial conflict. In general, a $n$th order conflict is a conflict that contains (at least) one of the trains in a $(n-1)$ th order conflict, but it is not a $(n-1)$ th order conflict itself. Figure 1 shows an example network with an initial conflict, first, second and third order conflicts. 


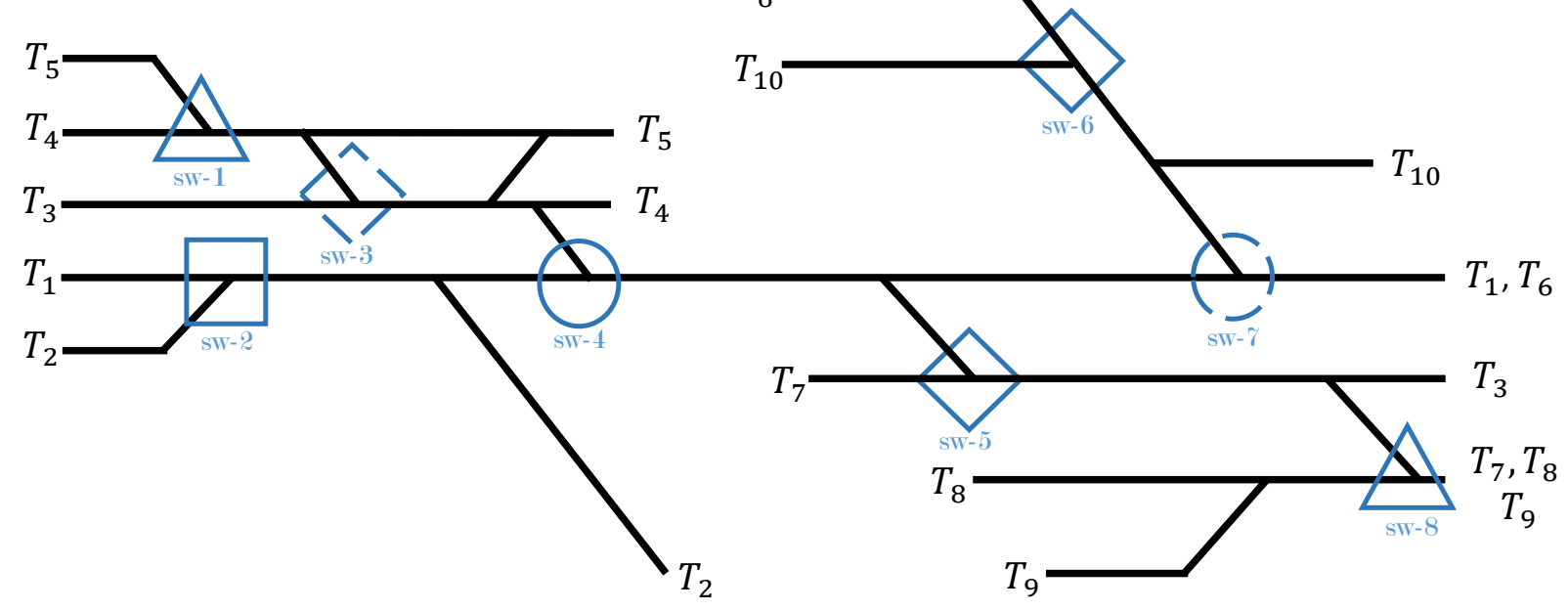

Figure 1: a fictive network with an initial conflict between trains $T_{1}$ and $T_{2}$ (indicated with a square) and new conflicts that might be considered in the dynamic impact zone. The routing of train $T_{i}$ is shown by putting the train's name in front and at the end of a line. All trains are assumed to drive from left to right. The circles represent first-order conflicts, the diamonds second-order and the triangles third-order conflicts. Full line shapes indicate the most likely conflicts (based on offline calculations). The names of the relevant switches are indicated below the shape.

The first train in the conflict is the train requiring the block section where the conflict takes place, first. The second train is then the train that wants to occupy the same block section second. A conflict can be resolved in two ways: delaying the first train or delaying the second train. The proposed DIZ heuristic examines both resolutions to the conflict by looking at a simplified progress of both trains during the next half hour. During this progress examination, new conflicts may arise, that will possibly also occur later on in real-time. In Figure 1, the square indicates the initial conflict. All other shapes are new conflicts, that could or could not be detected in the future. During this examination, the total delay of all considered trains is summed up. Then, the resolution attaining the least total delay is chosen and returned by the CPM.

Whenever a conflict is detected in real-time, the time required for determining the resolution and the time required for implementing the resolution has to be taken into account. Accordingly, the progress from the current situation during these times should not cause issues when implementing the resolution. Therefore, both the rerouting optimization and the DIZ heuristic start their calculations from the expected situation a control delay after the moment of detecting the conflict. Stated otherwise, no changes to ongoing operations 
can be executed during the duration of the control delay. Whatever happens within this time interval after detecting the conflict, cannot be changed by the conflict prevention module. Actually, this control delay gives the conflict prevention module this exact time to calculate (and implement) a resolution.

\section{Improvements and extensions}

This section introduces all improvements and extensions for making the CPS applicable for more relevant practical cases, which include larger and realistic networks, and to be solved faster. First, we consider rolling stock connections, required to deal with realistic networks and timetables, then four improvements making the CPS more efficient and (therefore) usable for larger networks, and finally two extensions especially relevant for railway operators:

1. Ensure rolling stock connections (Section 4.1)

2. Select more efficiently the conflicts and trains to consider in the DIZ (Section 4.2)

3. Solve conflicts in the DIZ in a smarter way than FCFS (Section 4.3)

4. Determining a distance limit for the space extension of the DIZ (Section 4.4)

5. Tackle areas with particular dispatching approaches (Section 4.5)

6. Consider passenger delays instead of train delays (Section 4.6)

7. Offer the possibility to cancel trains (Section 4.7)

Most of these improvements and extensions result from the (experimental) analysis of the approach presented in our previous paper (Van Thielen et al., 2018), and directly focus on the known open gaps and future research needs identified in that paper. In the previous paper we indicated, for instance, the drawback of resolving the conflicts in the dynamic impact zone by FCFS and that the computation time could be reduced by an even better selection of conflicts considered in the dynamic impact zone. This results now in improvements 2 and 3 (Sections 4.2 and 4.3). We also indicated that the time spent by the retiming/reordering part might grow rapidly in larger networks and that it would make sense to consider passenger flows when evaluating the progress of conflict resolutions. This results now in 4 and 6 (Sections 4.4 and 4.6).

\subsection{Rolling stock connections}

When planning the train lines and creating a timetable, also rolling stock connections are scheduled. Rolling stock connections are frequently used in practice to optimize the (capacity) usage of the rolling stock over different lines. While rolling stock connections are not per se innovative (other optimization approaches such as Pellegrini et al., 2016, 
Bettinelli et al., 2017 consider them), their industrial relevance, especially in large and densely used networks is large; we therefore extended our approach to include them.

Three different rolling stock connections are considered here: coupling, decoupling or making a turnaround. In order to address these connections, the CPS presented in Van Thielen et al. (2018) has to be adjusted. First, we look at the changes made in the rerouting optimization. Subsequently, we mention what has to be altered in the DIZ heuristic.

The rerouting optimization tries to find alternative routes within a station area. It is based on a flexible job shop problem and is solved with Cplex. During the optimization, only changes inside the station area are considered, not in the remainder of the network. This means that the lines on which all trains will enter and leave the station area and also the current position of the different trains already in the station area, are fixed. The rerouting starts from the expected situation in the station area, one minute after the moment of detecting the conflict (the control delay), whereas the end time is the latest departure time of one of the conflicting trains from the station area. The rerouting optimization considers all trains passing this station within this time period. As long as a train has not started its route in the station area, its planned route can be changed by the optimization.

\subsubsection{Mathematical model for rerouting}

The complete mathematical model used for rerouting is discussed in detail in our previous paper (Van Thielen et al., 2018), but we include it here with a brief discussion. Then we describe and motivate the changes made to the previous mathematical model.

\section{Parameters}

The parameters of the model describe the real-time situation of all trains in the station areas. 
$T=\left\{t_{1}, \ldots, t_{|T|}\right\}$, set of trains with $|T|$ the number of trains.

$R=\left\{r_{1}, \ldots, r_{|R|}\right\}$, set of routes with $|R|$ the number of routes.

$R_{t} \subset R=$ set of routes train $t$ can take.

$C=\left\{\left(\left(b_{i}, t_{i}, r_{i}\right),\left(b_{j}, t_{j}, r_{j}\right)\right) \mid b_{i}\right.$ and $b_{j}$ are block sections, sharing a part

of the infrastructure or that are identical, with $b_{i}$ belonging

to route $r_{i}$ from train $t_{i}$ and $b_{j}$ to route $r_{j}$ from train $\left.t_{j}\right\}$

$A=\left\{\left(t_{i_{1}}, \ldots, t_{i_{a}}, y\right) \mid\right.$ set of trains $\left(t_{i_{1}}, \ldots, t_{i_{a}}\right)$ having a connection of type $y$

$B_{p}=\left\{b_{p_{1}}, \ldots, b_{p_{n}}\right\}$, set of all block sections having a platform, with $n$ the number of platforms in the station area.

$\delta_{t}=$ earliest time instant at which train $t$ can start reserving the first block section in the station area.

$m r_{t, b}=$ minimum reservation time required for train $t$ before entering block section $b$

$m t_{t, b}=$ minimum travel time required for train $t$ to travel through block section $b$

$m c_{t, b}=$ minimum clearing time required for train $t$ to leave block section $b$

$m a_{t_{i}, t_{j}}=$ minimum association time between trains $t_{i}$ and $t_{j}$

$n b B S_{r}=$ total amount of block sections in route $r$ of this station area.

The subset $R_{t}$ includes routes that start and end at the predetermined signals of train $t$ when, respectively, entering and leaving the station area. Note that $\delta_{t}$ is the start reservation time instant determined by the timetable and the imposed delay on train $t$ at the current time. This model also uses block section 0, indicating the block section just before the station area. The sum of $m r_{t, b}, m t_{t, b}$ and $m c_{t, b}$ equals a block in the blocking time theory.

\section{Decision variables}

The decision variables are now introduced.

$$
x_{t, r}= \begin{cases}1 & \text { if train } t \text { takes route } r \in R_{t} \\ 0 & \text { otherwise }\end{cases}
$$

$S R_{t, b}=$ actual start reservation time of train $t$ on its $b$-th block section in the station area.

$D T_{t, b}=$ actual departure time of train $t$ from its $b$-th block section in the station area.

$A R_{t, b}=$ actual release time of train $t$ on its $b$-th block section in the station area.

$S D_{t}=$ secondary delay of train $t$ measured in this station area.

$P D_{t}=$ passenger delay of train $t$ measured in this station area. 
Note that $S R_{t, 1}$ differs from $\delta_{t}$ in the fact that $S R_{t, 1}$ can include additional delays due to conflicts in the station area. The index 0 in variable $x_{t, 0}$ corresponds to the current route of train $t$. The non-binary decision variables are expressed in seconds.

\section{Objective function}

As an objective function, a choice needs to be made between minimizing the secondary delays or the passenger delays. In case the secondary delays are minimized, the objective function becomes

$$
\operatorname{minimize} \sum_{t=1}^{|T|}\left(S D_{t}-\frac{x_{t, 0}}{K}\right) \text {, }
$$

with $S D_{t}=D T_{t, \mathrm{nbBS}_{r}}-\left(\delta_{t}+m r_{t, 1}+\sum_{b=1}^{\mathrm{nbBS}_{r}} m t_{t, b}\right)$.

\section{Constraints}

The mathematical model also requires constraints to assure that any solution is feasible. The first constraints assure the minimum duration according to the blocking time theory.

$$
\begin{gathered}
\qquad R_{t, 1} \geq \delta_{t}, \forall t \in T \\
\text { If } x_{t, r}==1 \text {, then } D T_{t, b-1} \geq S R_{t, b}+m r_{t, b}, \forall t \in T, b=1, \ldots, \operatorname{nbBS}_{r} \\
\text { If } x_{t, r}==1 \text {, then } D T_{t, b} \geq D T_{t, b-1}+m t_{t, b}, \forall t \in T, b=1, \ldots, \operatorname{nbBS}_{r} \\
\text { If } x_{t, r}==1 \text {, then } A R_{t, b} \geq D T_{t, b}+m c_{t, b}, \forall t \in T, b=1, \ldots, \operatorname{nbBS}_{r} .
\end{gathered}
$$

Every train in the model should use exactly one route:

$$
\sum_{r \in R_{t}} x_{t, r}=1, \forall t \in T
$$

Conflicts cannot appear in the optimal solution:

$$
\text { If } \begin{array}{r}
x_{t_{1}, r_{1}}+x_{t_{2}, r_{2}}==2 \text {, then } S R_{t_{1}, b_{1}} \geq A R_{t_{2}, b_{2}} \text { or } S R_{t_{2}, b_{2}} \geq A R_{t_{1}, b_{1}}, \\
\qquad \forall t_{1}, t_{2} \in T \text { and } \forall\left(\left(b_{1}, t_{1}, r_{1}\right),\left(b_{2}, t_{2}, r_{2}\right)\right) \in C .
\end{array}
$$

In other words: if train $t_{1}$ takes route $r_{1}$ and train $t_{2}$ takes route $r_{2}$ and these routes have conflicting block sections $b_{1}$ and $b_{2}$, then train $t_{1}$ cannot enter block section $b_{1}$ before train $t_{2}$ has released block section $b_{2}$ or vice versa. 


\subsubsection{Extending the mathematical model}

The rerouting optimization is now extended to take rolling stock connections between trains into account. Three different types of rolling stock connections are considered: coupling, decoupling and making a turnaround. Dependent on the type of connection, some additional constraints are imposed in the mathematical model.

Two trains sharing a connection at the given station area, should always use the same station platform.

$$
\text { If } b_{p} \in r_{i} \wedge b_{p} \in r_{j} \text {, then } x_{t_{i}, r_{i}}=x_{t_{j}, r_{j}}, \forall a=\left(T_{a}, y\right) \in A \text {. }
$$

If trains couple at a station platform, both trains should be allowed on the platform at the same time and they will depart from the platform as one train. Denote this train by $t_{k}$. The coupled train $t_{k}$ cannot depart before the two trains $t_{i}$ and $t_{j}$, that are coupled, both have arrived at the platform plus an additional service time $m a_{a}$ dependent on the association $a$. The block section including the platform is denoted by $b_{p}$. The block sections before and after the platform are denoted by $b_{p}-1$ and $b_{p}+1$ respectively.

$$
\begin{aligned}
& D T_{t_{k}, b_{p}} \geq D T_{t_{i}, b_{p}-1}+m a_{a}, \forall b_{p} \in B_{p}, t_{i}, t_{k} \in T, a=\left(t_{i}, t_{j}, t_{k},{ }^{\prime} \text { 'coupling' }\right) \in A, \\
& D T_{t_{k}, b_{p}} \geq D T_{t_{j}, b_{p}-1}+m a_{a}, \forall b_{p} \in B_{p}, t_{j}, t_{k} \in T, a=\left(t_{i}, t_{j}, t_{k},{ }^{\text {'coupling' }}\right) \in A,
\end{aligned}
$$

with $D T_{t_{k}, b_{p}}$ the departure time of train $t_{k}$ from block section $b_{p}$ and $A$ the set of all rolling stock connections.

If a train $t_{i}$ decouples at a station platform, two trains $t_{j}$ and $t_{k}$ will depart from the station. If these trains depart in opposite directions, it should only be imposed that $t_{j}$ and $t_{k}$ leave after the arrival of $t_{i}$ (including sufficient association time):

$$
\begin{aligned}
& D T_{t_{j}, b_{p}} \geq D T_{t_{i}, b_{p}-1}+m a_{a}, \forall b_{p} \in B_{p}, t_{i}, t_{j}, t_{k} \in T, a=\left(t_{i}, t_{j}, t_{k},{ }^{\prime} \text { decoupling' }\right) \in A, \\
& D T_{t_{k}, b_{p}} \geq D T_{t_{i}, b_{p}-1}+m a_{a}, \forall b_{p} \in B_{p}, t_{i}, t_{j}, t_{k} \in T, a=\left(t_{i}, t_{j}, t_{k},{ }^{\prime} \text { decoupling' }\right) \in A,
\end{aligned}
$$

If the trains depart in the same direction, the order in which trains depart is fixed based on how the trains are physically coupled. Assume that train $t_{j}$ departs first from the platform. In that case, the additional constraints are:

$$
\begin{gathered}
D T_{t_{j}, b_{p}} \geq D T_{t_{i}, b_{p}-1}+m a_{a}, \forall b_{p} \in B_{p}, t_{i}, t_{j}, t_{k} \in T, a=\left(t_{i}, t_{j}, t_{k}, \text { 'decoupling' }\right) \in A, \\
D T_{t_{k}, b_{p}}>D T_{t_{j}, b_{p}}, \forall b_{p} \in B_{p}, t_{j}, t_{k} \in T, a=\left(t_{i}, t_{j}, t_{k}, \text { 'decoupling' }\right) \in A .
\end{gathered}
$$

In case a train $t_{i}$ has a turnaround at its terminal station, it is necessary that $t_{i}$ remains occupying the platform until the connected train $t_{j}$ takes over. Therefore train $t_{j}$ should start reserving the block section immediately when $t_{i}$ releases the block section. However, 
the earliest possible departure of $t_{j}$ is dependent on both the timetable and the minimum time required for making a turnaround.

$$
\begin{array}{r}
A R_{t_{i}, b_{p}}=S R_{t_{j}, b_{p}}, \forall b_{p} \in B_{p}, t_{i}, t_{j} \in T, a=\left(t_{i}, t_{j}, \text { 'turnaround' }\right) \in A, \\
D T_{t_{j}, b_{p}} \geq S R_{t_{j}, b_{p}}+m t_{t_{j}, b_{p}}+m a_{a}, \forall b_{p} \in B_{p}, t_{i}, t_{j} \in T, a=\left(t_{i}, t_{j}, \text { 'turnaround' }\right) \in A,
\end{array}
$$

with $A R_{t_{i}, b_{p}}$ the release time of train $t_{i}$ on block section $b_{p}, S R_{t_{j}, b_{p}}$ the reservation time of train $t_{j}$ on the same block section and $m t_{t_{j}, b_{p}}$ the minimum travel time of train $t_{j}$ for block section $b_{p}$. This approach to deal with rolling stock connections can also be used to ensure guaranteed passenger transfers. Due to a lack of data on passenger flows for the considered study area, this is not included in this paper.

\subsubsection{Rolling stock connections in the heuristic}

In the DIZ heuristic, the rolling stock connections are guaranteed by imposing the same conditions, as mentioned above for the rerouting optimization, when evaluating the progress of the different trains.

\subsection{Updating the potentially conflicting trains}

The dynamic impact zone is based on the offline-determined set of most likely conflicts and potentially conflicting trains. The set of potentially conflicting trains are trains using the same infrastructure within a time frame of 20 minutes. However, at a particular time, some potentially conflicting trains can still be on schedule or significantly delayed, and therefore not actually be potentially conflicting anymore. Therefore, their current timing, including all known delays up to the time instant of detection of the initial conflict, are considered when determining whether the offline-determined potentially conflicting trains should actually still be considered as such or not. Including less trains in the progress examination will reduce the computation time for the DIZ heuristic. This feature is illustrated with an example.

\section{Example}

Figure 1 shows a small network where ten trains are driving from left to right. The square indicates the initial conflict detected by TMS. All other conflicts are potential conflicts, i.e. conflicts between potentially conflicting trains. For every potential conflict, it is evaluated whether this conflict should be included in the dynamic impact zone. A potential conflict becomes a new conflict if it is detected during the progress examination. The corresponding blocking times of all trains on the switches indicated are stated in Table 1. These blocking times include all delays known up to the current time instant, i.e. the time instant that the initial conflict is detected.

The initial conflict is detected at 06:55:00. All new conflicts are situated in the future. Looking at the current situation, only one conflict in addition to the initial conflict will actually take place in the future: $T_{1}$ and $T_{3}$ will conflict on $\mathrm{sw}_{4}$. 
Previous research did not include a real-time update of the potentially conflicting trains. The impact zone was only limited by the time limit of the progress examination (i.e. 30 minutes) and the order of the potential conflicts. When looking at Figure 1, previously all new conflicts with a circle and diamond with a full line (all first-order new conflicts and most likely second-order new conflicts) were considered in the dynamic impact zone. Trains $T_{1}, T_{2}, T_{3}, T_{6}, T_{7}$ and $T_{10}$ were taken into account during the progress examination. However, based on the current information on all trains, it is now checked which trains still have a potential conflict within a time range of $\gamma$ minutes. A value of 5 minutes is used for $\gamma$ in this example. First, the first-order conflicts are looked upon. Clearly, the conflict between $T_{1}$ and $T_{6}$ should not be considered. Therefore, $T_{6}$ (and $T_{10}$ ) will not be included in the dynamic impact zone. Also, the conflict between $T_{3}$ and $T_{7}$ is not considered. The set of trains in the dynamic impact zone is now limited to $T_{1}, T_{2}$ and $T_{3}$. So, a little more computation time is invested in updating the set of potential conflicts, but this should save more computation time when considering the conflicts during the progress examination and lead to (at least) the same quality of results. The effect, both on calculation time and quality of the results, will be illustrated in Section 5 .

\begin{tabular}{c|c|c|c|c}
\hline$T_{a}$ & $T_{b}$ & switch & Start-end of blocking time $T_{a}$ & Start-end of blocking time $T_{b}$ \\
\hline$T_{1}$ & $T_{2}$ & $\mathrm{sw}_{2}$ & $07: 01: 00-07: 02: 30$ & $07: 00: 30-07: 01: 15$ \\
\hline$T_{1}$ & $T_{3}$ & $\mathrm{sw}_{4}$ & $07: 04: 00-07: 05: 00$ & $07: 04: 30-07: 05: 45$ \\
\hline$T_{1}$ & $T_{6}$ & $\mathrm{sw}_{7}$ & $07: 10: 30-07: 12: 00$ & $07: 20: 30-07: 22: 15$ \\
\hline$T_{3}$ & $T_{4}$ & $\mathrm{sw}_{3}$ & $07: 02: 15-07: 03: 45$ & $07: 07: 45-07: 08: 45$ \\
\hline$T_{3}$ & $T_{7}$ & $\mathrm{sw}_{5}$ & $07: 15: 00-07: 16: 45$ & $07: 08: 00-07: 09: 45$ \\
\hline$T_{4}$ & $T_{5}$ & $\mathrm{sw}_{1}$ & $07: 06: 15-07: 08: 00$ & $07: 04: 00-07: 05: 00$ \\
\hline$T_{6}$ & $T_{10}$ & $\mathrm{sw}_{6}$ & $07: 15: 45-07: 17: 45$ & $07: 01: 15-07: 03: 00$ \\
\hline$T_{7}$ & $T_{8}$ & $\mathrm{sw}_{8}$ & $07: 13: 45-07: 15: 00$ & $07: 21: 45-07: 23: 00$ \\
\hline
\end{tabular}

Table 1: Blocking times on the switches where a potential conflict is situated on the example network in Figure 1.

\subsection{Dealing with new conflicts in the heuristic}

In the algorithm described in (Van Thielen et al., 2018), we assumed during the progress examination that the new conflicts were resolved according to First Come, First Served (FCFS). A more accurate way of resolving these conflicts is now presented here. This improves the estimation of the total expected delay, and therefore also the performance of the DIZ heuristic.

Within the progress examination of the heuristic, new conflicts are detected and need to be resolved. Evidently, it is computationally not possible to consider all conflict resolution combinations in real-time. However, the resolution of this new conflict is directly affecting 
the decision made in the DIZ heuristic because the decision is partly based on the delay caused by resolutions of these new conflicts. Previously, new conflicts were resolved by using FCFS, because it is fast. Now, little additional computation time is spent on a smarter way to resolve these new conflicts in order to improve the quality of the heuristic.

Whenever a new conflict is detected during the progress examination of the heuristic, it is now determined what the immediate impact is of delaying one of the two trains in terms of train delay. A small example illustrates what we call the immediate impact. Figure 2 shows the routes of three trains within a progress examination of the heuristic. The path of $T_{1}$ is indicated by the red line, the path of $T_{2}$ by the green line and the path of $T_{3}$ by the purple line. During the progress examination of the heuristic, a new conflict is detected between $T_{1}$ and $T_{2}$ on block section BS-3. In order to determine which train to delay, the immediate impact in terms of train delay on both trains is determined first. Trains $T_{1}$ and $T_{2}$ share infrastructure on several subsequent block sections, i.e. BS-3, BS-4, BS-5 and BS-7. This implies that $T_{1}$ and $T_{2}$ will be driving behind each other on all these block sections. Moreover, $T_{1}$ might be delayed due to a conflict with $T_{3}$ on BS-7 and then further delay $T_{2}$, if $T_{1}$ drives before $T_{2}$. Therefore, in this case, it might be better to let $T_{2}$ go first. More generally, if one train in a conflict, $T_{1}$, is allowed to drive first on a common part of the infrastructure and is expected to be delayed due to another new conflict later on this common part, then the other train in the conflict, $T_{2}$, will be additionally delayed. Therefore, it would be better to let $T_{2}$ go first. This is what we call considering the immediate impact when deciding on how to resolve a new conflict.

Specifically, we first determine the set of subsequent block sections with some part of the infrastructure in common belonging to the two trains ( $T_{1}$ and $T_{2}$ in the example) in the new conflict. Then, for every block section in this set (BS-3, BS-4, BS-5 and BS-7 in the example), we look at any other train in the dynamic impact zone that runs on some common infrastructure ( $T_{3}$ in the example). If its occupancy based on the delay characteristics at the detection time of the new conflict (partly) coincides with the time interval of one of the two trains in the new conflict, then a delay is imposed on the train in the new conflict ( $T_{1}$ in the example). This delay is then added to the delay resulting from resolving the new conflict. The option with the least total delay is chosen.

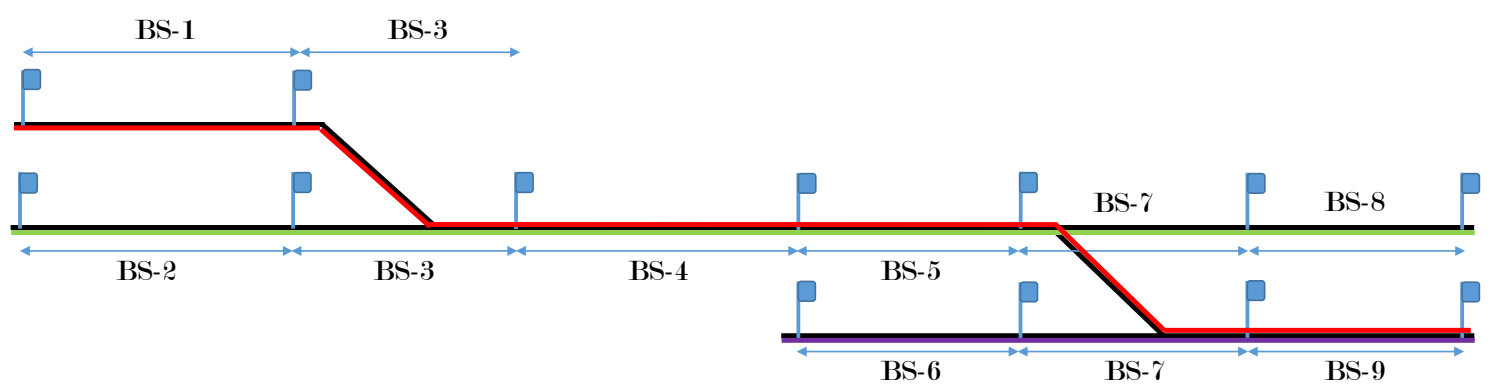

Figure 2: Three train paths on a small network: the red line indicates the path of $T_{1}$, the green line the path of $T_{2}$ and the purple line the path of $T_{3}$. 


\subsection{Limiting the geographical size of the DIZ}

In this section, a new parameter is introduced that allows the DIZ heuristic to be applied to larger networks while limiting the computation time. Until now, the dynamic impact zone was only limited by the duration of the progress examination. When considering large and complex networks, this limitation could be insufficient to keep the computation time low. Therefore, we will now also limit the dynamic impact zone by considering a maximal distance. Specifically, conflicts will only be included in the dynamic impact zone if they are situated less than $\epsilon \mathrm{km}$ in track distance away from the initial conflict.

Figure 3 shows the small network from Section 4.2. There is an initial conflict between $T_{1}$ and $T_{2}$. All conflicts in the corresponding dynamic impact zone within $\epsilon=25 \mathrm{~km}$ are indicated in red. Compared to the standard DIZ heuristic, there are less trains and conflicts considered in the new dynamic impact zone. With $\epsilon=25 \mathrm{~km}$, only trains $T_{1}, T_{2}$, $T_{3}$ and $T_{7}$ are taken into account during the progress examination of the heuristic. This should speed up the calculations, hopefully without reducing the quality of the solution too much. This parameter is different from setting a time limit in the progress examination because speed patterns are accounted for in the blocking times. Therefore, every train has a specific time for traveling through a block section. The effect of introducing $\epsilon$ will be tested in Section 5.
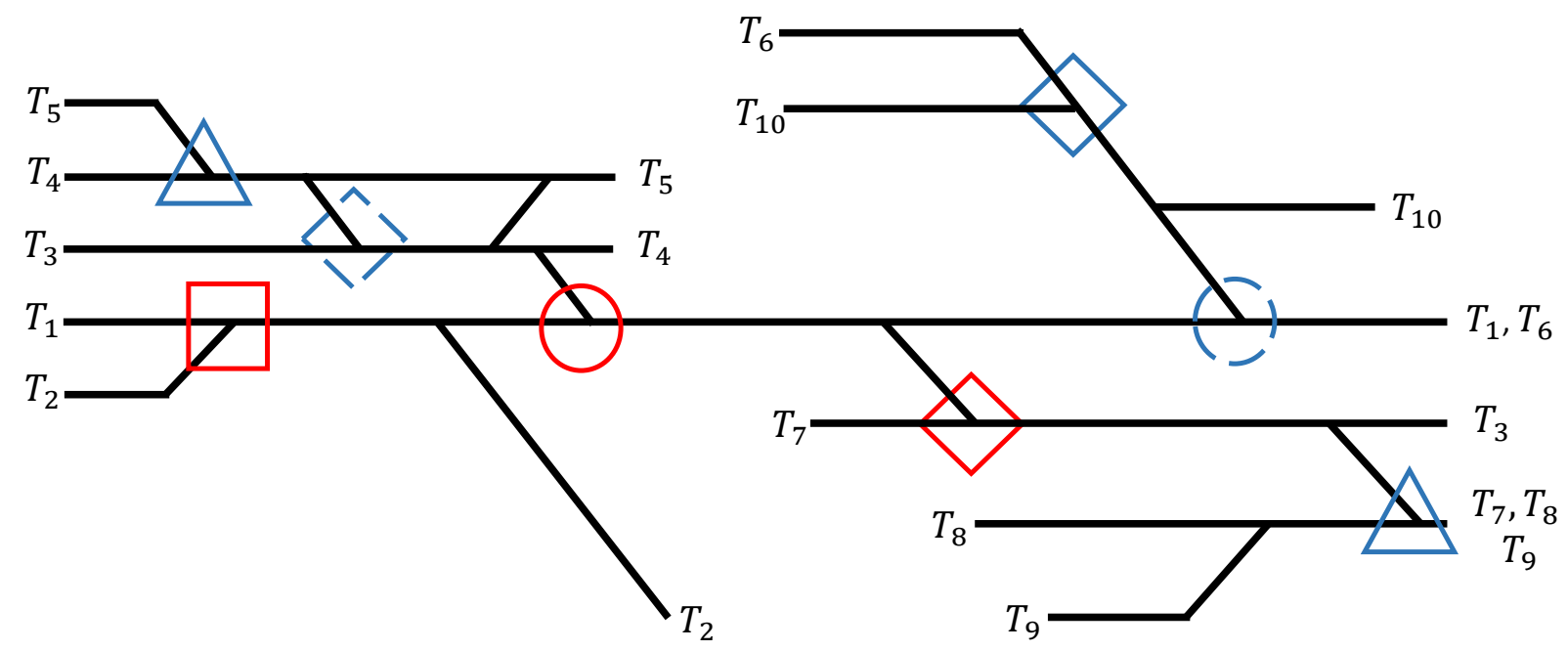

Figure 3: Small network with different conflicts indicated by a geometric shape. Only the first and second-order most likely conflicts within a distance of $\epsilon=25 \mathrm{~km}$ are considered in the dynamic impact zone (indicated in red). 


\subsection{Areas with particular dispatching approaches}

A specific industrial constraint is that some parts of the network have a specific set of business rules which are hard, when not impossible to be modeled in the optimization scheme; therefore, they should be modeled as having their own particular dispatching approach.

This is typical for complex areas, in our case corresponding to station areas integrating multiple very close stations, deeply interconnected, such that a decision at some point in time affects strongly traffic in all other parts of the station area. An example case in the Belgian network is the area around Antwerpen Centraal, shown in Figure 4. In this area, there can be made no clear distinction between the switch areas and the platform areas. In addition, the blocking time theory is applied differently in these areas, because trains have a limited speed and the block sections are smaller. In busy periods, trains might even drive 'on sight' instead of based on the available signals. It makes no sense to apply our CPS to such an area. Moreover, it would require an even more detailed timetable and infrastructure data to run our simulation in a realistic way. Therefore, this small part is considered as a black box during simulation and during our CPS. This means that the behavior of the trains in that area is considered fixed, i.e. this cannot be influenced by our CPS. Based on when and where each train enters this area it is fixed when and where it will leave the area (with or without an additional delay).

In the network considered in this paper, the red rectangle in Figure 4 will be considered as a black box area. The station of Antwerpen-Centraal has 3 levels, serving in total 14 platforms. Trains coming from the south will be monitored until the station of Antwerpen-Berchem and trains coming from the north are monitored until Antwerpen-Oost or Antwerpen-Centraal. Trains are assumed to keep driving according to their situation before entering the black box area, implying that every train delay will remain, while running through the area. Also, rolling stock connections made at Antwerpen-Centraal are assumed to be retained. 


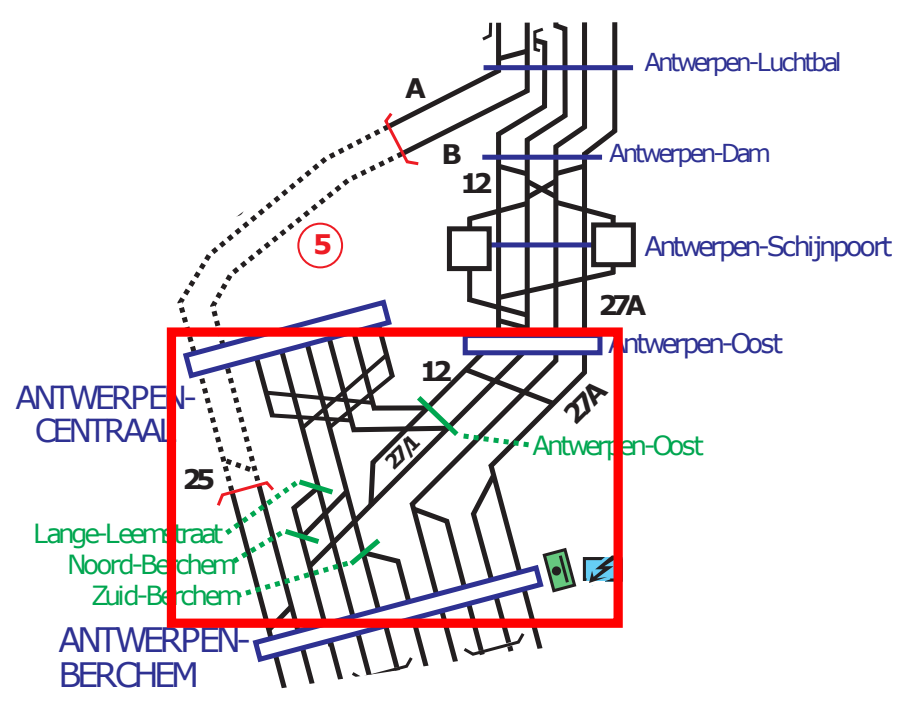

Figure 4: A close-up of a schematic representation of the area surrounding the station of Antwerpen-Centraal.

\subsection{Passenger vs train delays}

After including some important adjustments and improvements to the CPM, we discuss the practical and industrial relevance of two extension; the first one pertains the objective function, introducing the possibility to minimize passenger delays; the second one the possibility to cancel trains, if circumstances so dictate.

Infrastructure managers are typically interested most in minimizing the train delays, whereas railway operators might want to minimize the passenger delays. Previously, the CPS only considered train delays. Nonetheless, there is a clear trend in including passenger aspects in the traffic control. Therefore the CPS is adapted in such a way that it can minimize passenger delays instead of secondary train delays. With this section, we also want to illustrate how easy it is to adapt our method to another objective function. Actually, only the objective needs to be changed while all the rest remains exactly the same. We consider this as a strength of our method that the objective can be easily adapted to the requirements of the user.

In absence of richer behavioral models, the passenger delay equals the amount of a delay times the number of passengers affected by that delay. The total passenger delay is then the sum over all passengers arrived in the study area times their arriving delay plus all passengers leaving the network times the delay of the train when leaving the study area. During the progress evaluation of the DIZ heuristic, the passenger delay is evaluated instead of the train delay. Based on the specific input and the current situation in belgium, freight trains or empty rides are assumed to have no passengers. In order to still take them into account, every freight train is assumed to have one passenger aboard, implicating that the passenger delay equals the train delay. It would be straightforward to use other weights for freight trains or empty rides, depending on the importance they should get when 
passenger delays are used as the objective. In this case, the smallest weight is used for the freight trains since in Belgium, in practice, freight trains typically get the lowest priority, certainly during rush hours. Since empty rides have no passengers, they are also given the lowest weight when passenger delays are used as an objective. These empty rides typically also have a much less strict timetable. It should also be noted that if a train is currently empty, but passengers will board it in one of its next stations, it is not considered as an empty ride. As mentioned above, the weight that will be used in this case is the number of passengers affected. Sensitivity to different classes and the tradeoff between performance for some classes versus performance for the entire system have been for instance described in Luan et al (2016).

In the rerouting optimization, the objective is altered to minimizing passenger delays as follows. Let $P D_{t}$ be the passenger delay of train $t$. The passenger delay is measured as the delay of a train times the number of passengers affected. The number of passengers affected is determined by taking the average number of passengers on the train right before and after the stop at the current station, which is denoted by $P N_{t}$. The objective function then becomes

$$
\text { minimize } \sum_{t=1}^{|T|}\left(P D_{t}-\frac{x_{t, 0}}{100}\right),
$$

with $P D_{t}=P N_{t} \times S D_{t}$ where $S D_{t}$, defined at Equation (1), equals the secondary delay of train $t$ attained in this station area during the considered time horizon.

The second term of the objective function, with $x_{t, 0}$, assures that the original route from the train is preferred if the passenger delay cannot be decreased. This is similar to the original objective function.

\subsection{Canceling passenger trains}

If delays become longer, it can be beneficial to cancel a train because the train will cause too many more conflicts in the remainder of its route, and passengers will anyway experience a longer travel time comparable with the delay of the train. In networks with high frequent services, some delay might be larger than the headway, really putting in doubt whether such delayed train should use capacity in the network and cause further knock on effects, or instead it is not better to tolerate a cancellation of a train to allow a faster recovery of the delay. Considering this option was also asked by the Belgian Infrastructure manager (Infrabel). Also in the current practice, cancelling a train is considered an option, if this helps to avoid many other knock-on delays and allows to reuse the rolling stock again according to the timetable (for instance by canceling a train for the end of its current trip and the first part of its next trip).

Therefore, the set of possible control actions available to the DIZ heuristic is extended such that a maximum of four possibilities are regarded: delay the first train, delay the second train, cancel the first train, cancel the second train. In this paper, only passenger trains are allowed to be canceled. Trains can only be canceled at a station platform. If the conflict does not take place at a station platform, then the train needs to be delayed 
first to resolve the conflict. The train can then be canceled at the next station where all passengers can disembark, and the train can be put on a side track.

Whenever a train is canceled, its (remaining) rolling stock connections are checked. In case of decoupling, the two, new trains are also canceled because the first train was canceled. In case of a turnaround at a terminal station, it is examined whether the second train should pass the station where the first train was canceled. If this is the case, then the second train is only canceled up to this station. The second train is then assumed to depart again, at its scheduled time in this station, if this is feasible. If not, it will depart with a delay.

Canceling a train certainly causes annoyance to passengers, and is a measure that is considered worse than delaying a train. Therefore, canceling a train will be evaluated by a penalty delay of $\delta$ minutes. In Section 5, the effect of altering this parameter from 30 to 60 minutes is examined. This is comparable with the headway of the trains, i.e. a train can be canceled only if it would arrive after the next planned service on the same line, and if it further improves the overall delay in the network.

\section{$5 \quad$ Experimental results}

In this section, we first discuss the simulation framework that allows us to evaluate our CPS and the experimental setup. Then we explain how the experiments are designed in order to investigate in a systematic way the different modifications of the CPS. This is then applied to the first case study. Finally, the best found CPS is applied to an even larger network, including an area with a particular dispatching strategy.

\subsection{Simulation framework}

The performance of the CPS is evaluated by integrating it in a simulation framework. This framework is identical to the one used in Van Thielen et al. (2018). This simulation framework consists of four main components that model a complete TMS interacting with real life. The four components are a simulator module, a conflict detection module, a Conflict Prevention Module (CPM) and a dispatcher or automated machine. Figure 5 shows how these components interact. The discrete event simulation resembles the realtime movements of all trains. Within the conflict detection module, train movements are predicted for a prediction horizon ahead and conflicts are detected, resembling an advanced TMS. The CPM includes the Conflict Prevention Strategy (CPS) discussed in this paper (called iCPS) with a rerouting optimization and the retiming heuristic (DIZ heuristic), in order to determine the best resolution for the conflict. In the dispatcher module, we assume that an automated machine accepts all resolutions sent by the CPM. For more information on this closed loop simulation setup, we refer the interested reader to Van Thielen et al. (2018). 


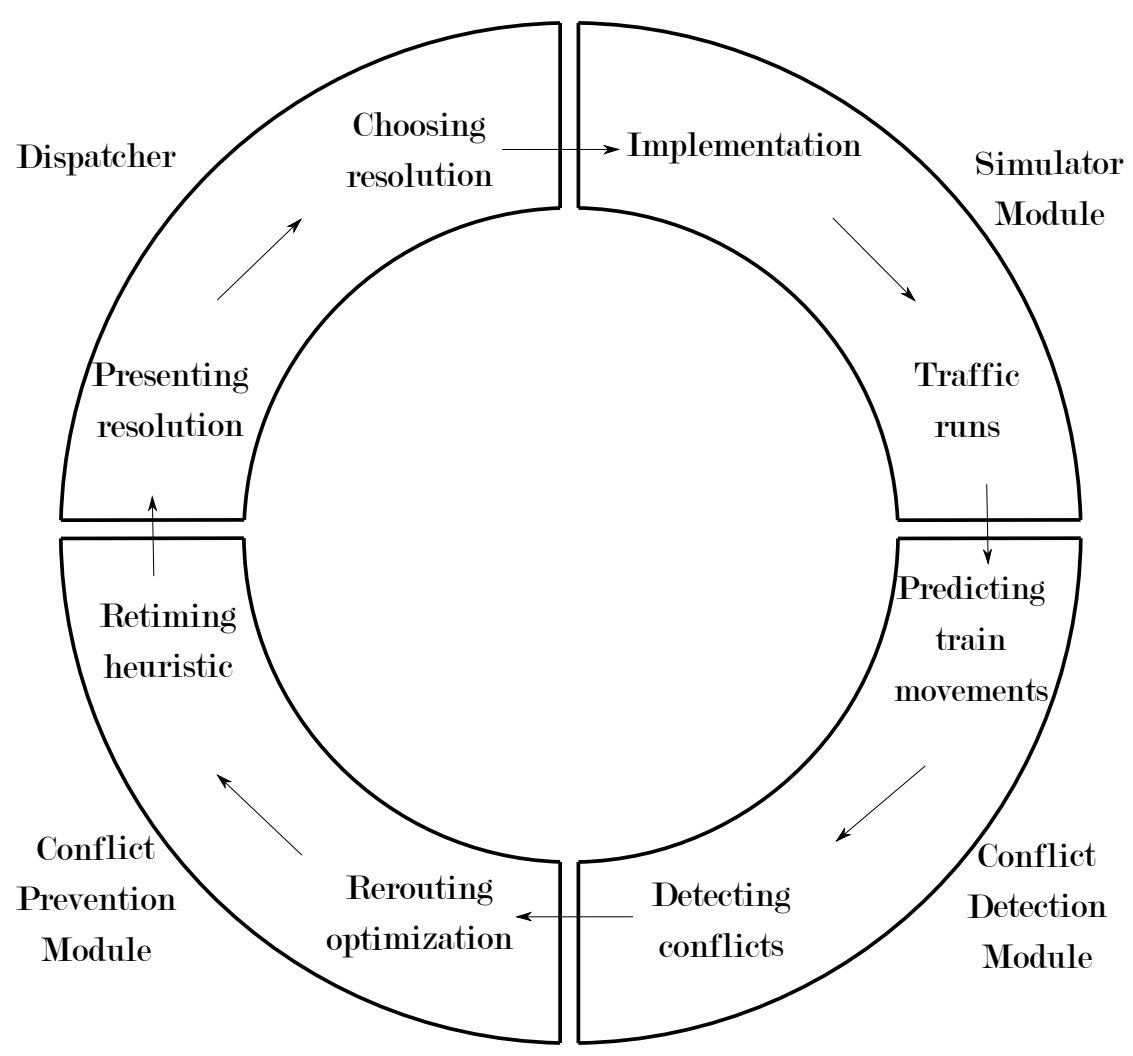

Figure 5: Schematic overview of the closed loop framework with its different components.

\subsection{Experimental setup}

The new CPS, called iCPS, is tested and evaluated in this section. We start by describing the performance of the iCPS, including also sensitivity to the components introduced compared to previous research. In particular, we address first the absolute quality improvement of the iCPS, considering rolling stock connections constraints, with regards to common benchmarks, including the extension of Van Thielen et al. (2018) to the case of rolling stock connections constraints. The most basic dispatching strategy considered as benchmark is First Come, First Served (FCFS), where the train first wanting to reserve the block section where the conflict occurs, is allowed to drive first. This strategy resembles an inexperienced dispatcher. As a third and last comparison, the first-order heuristic (including rerouting) is considered. This heuristic is also based on a progress examination during the next half hour, but the impact zone is limited to first-order conflicts. This strategy thus makes no use of offline calculations or any improvement/extension discussed in this paper, but it does consider rolling stock circulation.

Then we study the benefit of the algorithmic components introduced in this paper, namely the more efficient selection and resolution of the conflicts and the limitation to the size of the dynamic impact zone. We then discuss the impact of considering passengers delays as objective and of considering the cancellation of trains. Finally, an even larger 
test case is presented and we show the interoperability of the iCPS with other dispatching algorithms.

In order to evaluate the different components of the iCPS, the prediction horizon in the conflict detection module is set to the standard 5 minutes, the heuristic horizon of the iCPS to 30 minutes and the control delay to 60 seconds (see Van Thielen et al. (2018) for more information).

The offline calculations are based on 350 runs from a delay scenario with $\alpha \%$ of the trains delayed. The value $\alpha$ is randomly taken from the interval [20\%,80\%]. For each version of iCPS, 20 runs from a delay scenario $\alpha$ are taken, where $\alpha$ is randomly taken from the interval $[20 \%, 80 \%]$. In one run, approximately $\alpha \%$ of all trains, which are randomly chosen, are given a random delay from an exponential distribution with an average of 3 minutes and a maximum of 15 minutes. The computation time of the rerouting optimization is limited to 30 seconds.

In these experiments, the iCPS is evaluated based on the total secondary train delay, the total passenger delay and the computation time required. The total train delay sums up the secondary delays of all trains at the end of the simulation horizon or when leaving the study area. The total passenger delay looks at the delay at every station times the number of passengers that left the train at that station. If the train leaves the study area, the passenger delay equals the delay when leaving the study area times the maximum number of passengers on the train for the remainder of the train's route. In the case of a freight train or an empty ride, the passenger delay is set equal to the train delay.

Due to a lack of realistic data, simple assumptions are made on the passenger flows. Trains can either be busy or quiet. A busy train has approximately four times the number of passengers aboard compared to a quiet train. The passengers function is a stepwise function that changes randomly in every station. It is assumed that the number of passengers increases until the middle point of the train's full route. After the middle point of its route, the number of passengers decreases. Figure 6 depicts the passenger pattern of a quiet train. 


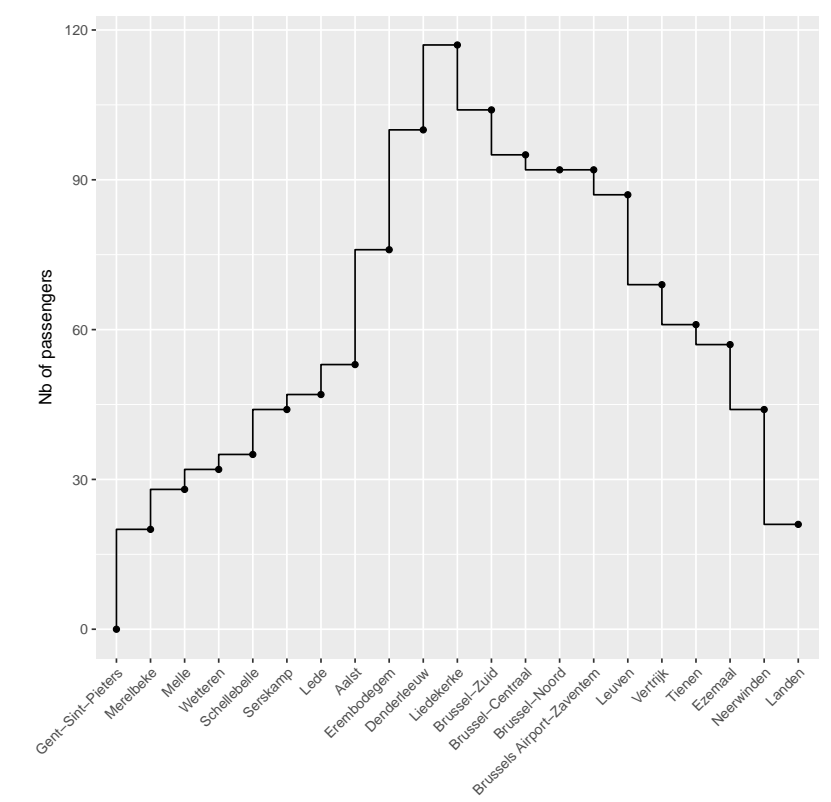

Figure 6: The passenger pattern of a quiet train for its full route.

\subsection{Case Study 1}

The iCPS and benchmarks are extensively tested on a large and complex study area including the provinces of East and West Flanders in Belgium (see Figure 7). This microscopic network contains 130 stations and 11766 block sections. Both freight and passenger trains are taken from the microscopic timetable of $17 / 03 / 2017$. The simulation horizon is set from 7 a.m. to 8 a.m. During this time horizon and in this study area, there are 240 trains considered. All 51 rolling stock connections taking place between these trains in practice, i.e. (de)coupling and turnarounds, are taken into account. 


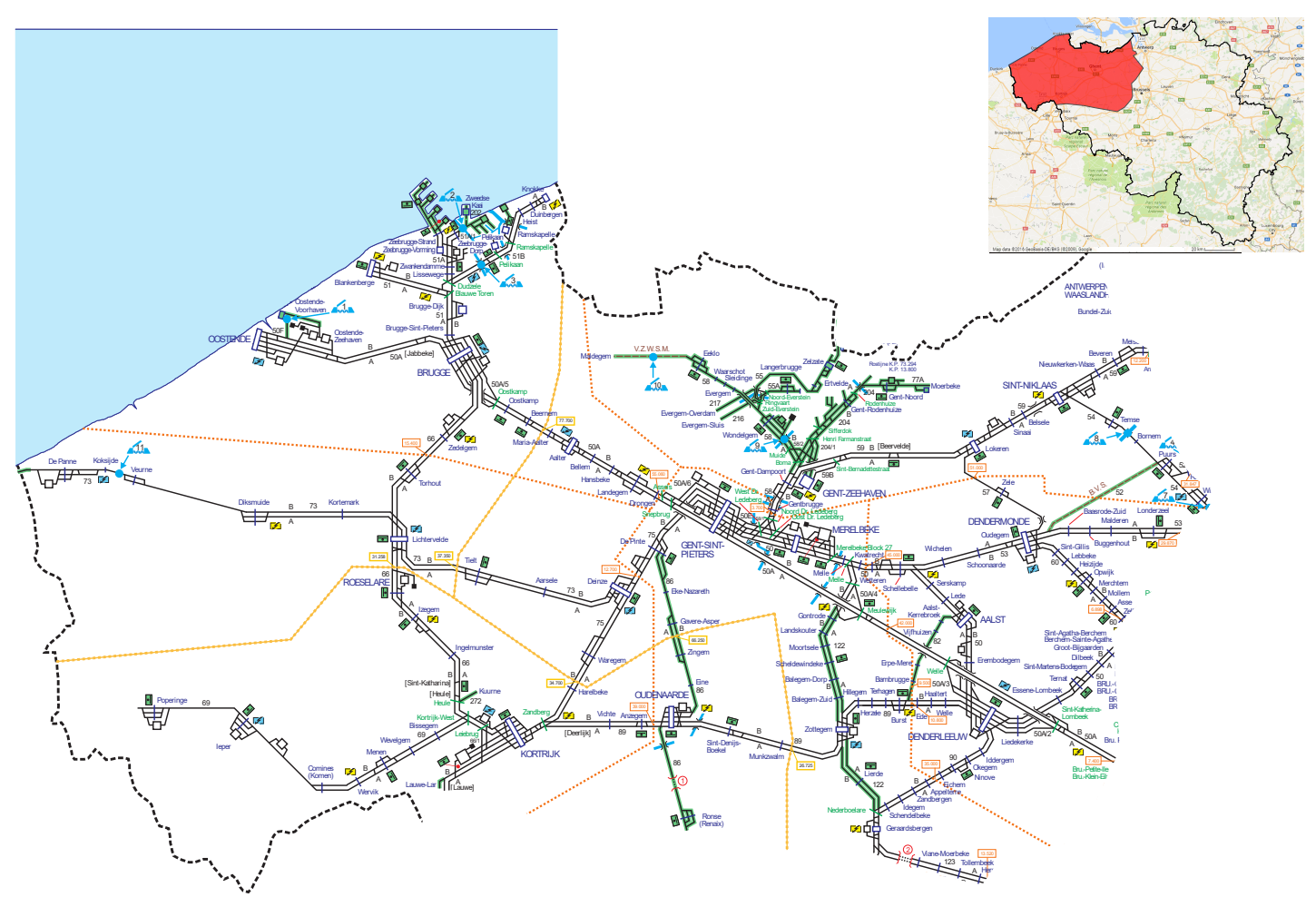

Figure 7: Study area: the provinces of West and East Flanders in Belgium.

\subsection{Performance of iCPS}

The best performing CPS, iCPS, uses a dynamic impact zone with a distance limitation of $50 \mathrm{~km}$ and all algorithmic improvements presented in this paper. In this section we evaluate the performance of this iCPS. We show in Table 2 the results in terms of secondary train delays (Train D), passenger delays (Pass D) and average and maximum computation time of the proposed iCPS. Concerning these average and maximum computation times, it should be noticed that in about $15 \%$ of the times the rerouting is applied, it also reaches its time limit, regardless of the algorithmic configuration (more details in Van Thielen (2019)). Rolling stock connections are taken into account for all approaches. We include as benchmarks the extension of Van Thielen et al. (2018) to consider the rolling stock connections, the FCFS, and the First order heuristic. It should be noted that passenger delay is reported here, but it is not used as objective function.

Between brackets is the improvement compared to the FCFS strategy. The iCPS strategy clearly outperforms FCFS and the first-order heuristic both on secondary train delays and passenger delays, and achieves a further improvement compared to the extension of Van Thielen et al. (2018). The computation time of the iCPS for a single initial conflict includes the creation and execution of the rerouting optimization, the creation of the 
dynamic impact zone and the execution of the DIZ heuristic itself. Here as well, iCPS achieves a further improvement compared to the extension of Van Thielen et al. (2018).

\begin{tabular}{c|c|c|c|c}
\hline Strategy & $\begin{array}{c}\text { Train D } \\
\text { (in min) }\end{array}$ & $\begin{array}{c}\text { Pass D } \\
\text { (in min) }\end{array}$ & $\begin{array}{c}\text { Avg comp } \\
\text { time (in s) }\end{array}$ & $\begin{array}{c}\text { Max comp } \\
\text { time (in s) }\end{array}$ \\
\hline FCFS & 740 & 158768 & $<0.1$ & $<0.1$ \\
First-order & $416(-43.8 \%)$ & $126171(-20.5 \%)$ & 2.2 & 37.6 \\
Van Thielen et al. (2018) & $383(-48.2 \%)$ & $127444(-19.7 \%)$ & 2.7 & 34.1 \\
iCPS & $359(-51.5 \%)$ & $123268(-22.3 \%)$ & 2.2 & 34.1 \\
\hline
\end{tabular}

Table 2: Comparison of the proposed iCPS, including rolling stock connections.

\subsection{Impact of the algorithmic improvements}

Table 3 shows the results of including the improvements from Sections 4.2 and 4.3. The first line presents again the results for the extension of Van Thielen et al. (2018). The second line includes the update of the potential conflicts during the creation of the DIZ. The next line shows the effects of the smart manner of resolving new conflicts during the progress examination. The fourth line combines the two previous methods. In order to make a fair evaluation of these improvements, none of the methods in this table include the distance limit discussed in the next section. This also explains why the results for the last line are slightly different compared to the last line in the previous table. Including a new way to deal with conflicts decreases the total train delays with approximately $6.8 \%$ compared to the strategy of Van Thielen et al. (2018) (including rolling stock circulation). The computation time only decreases slightly, but effects of this improvement are assumed to be larger on larger networks.

\begin{tabular}{c|c|c|c|c|c}
\hline $\begin{array}{c}\text { Update } \\
\text { of conflicts }\end{array}$ & $\begin{array}{c}\text { Smart conflict } \\
\text { resolution }\end{array}$ & $\begin{array}{c}\text { Train D } \\
\text { (in min) }\end{array}$ & $\begin{array}{c}\text { Pass D } \\
\text { (in min) }\end{array}$ & $\begin{array}{c}\text { Avg comp } \\
\text { time (in s) }\end{array}$ & $\begin{array}{c}\text { Max comp } \\
\text { time (in s) }\end{array}$ \\
\hline- & - & 383 & 127444 & 2.7 & 34.1 \\
$\checkmark$ & - & $370(-3.3 \%)$ & $126890(-0.4 \%)$ & 2.3 & 33.6 \\
- & $\mathbb{J}$ & $357(-6.8 \%)$ & $122166(-4.1 \%)$ & 2.6 & 34.0 \\
$\sqrt{ }$ & $\mathbb{\checkmark}$ & $358(-6.8 \%)$ & $122107(-4.2 \%)$ & 2.5 & 34.0 \\
\hline
\end{tabular}

Table 3: Results of the improvements in the dynamic impact zone based on train delays and computation time.

\subsection{Impact of space limitation}

Table 4 shows results for different parameter settings regarding the size of the impact zone, in increasing sizes. The first line corresponds to our iCPS, but without a distance limit, 
also reported on the last line of the previous table (Table 3). All approaches reported here include the algorithmic improvements examined in the previous section.

\begin{tabular}{|c|c|c|c|c|}
\hline $\begin{array}{c}\text { Size of the } \\
\text { DIZ }\end{array}$ & $\begin{array}{l}\text { Train D } \\
\text { (in min) }\end{array}$ & $\begin{array}{l}\text { Pass D } \\
\text { (in min) }\end{array}$ & $\begin{array}{l}\text { Avg comp } \\
\text { time (in s) }\end{array}$ & $\begin{array}{l}\text { Max comp } \\
\text { time (in s) }\end{array}$ \\
\hline unlimited & 358 & 122107 & 2.5 & 34.0 \\
\hline $10 \mathrm{~km}$ & $379(+5 \%)$ & $126534(+3.6 \%)$ & 1.8 & 33.7 \\
\hline $50 \mathrm{~km}$ & $359(+0.3 \%)$ & $123268(+1.0 \%)$ & 2.2 & 34.1 \\
\hline $100 \mathrm{~km}$ & $359(+0.3 \%)$ & $123196(+0.9 \%)$ & 2.3 & 34.2 \\
\hline
\end{tabular}

Table 4: Comparison of performance with different distance limitations for iCPS.

Setting a maximum distance influences both the quality and the computation time of the solution. If the maximum distance is set to 50 or $100 \mathrm{~km}$, the results are similar to the ones without setting a maximum distance. Lowering the maximum distance to $10 \mathrm{~km}$ gives a notable reduction in computation time, though the train delays increase significantly. Therefore, we consider the distance limit of $50 \mathrm{~km}$ as the best result and incorporated this in our final iCPS reported in Table 2 as well. It should be noticed here that this distance limit becomes even more important when the network considered would become much larger. For those networks, this distance limit will allow to obtain the same quality results, without increasing the computation time.

The average number of new conflicts considered in the dynamic impact zone is shown in Table 5. This comparison should be regarded carefully, because a certain conflict resolution might lead to more or less initial conflicts to be detected later. Clearly, updating the potential conflicts (see Section 4.2) and smartly resolving the potential conflicts (see Section 4.3 ), resulting in iCPS with unlimited size of DIZ, has a strong effect on the size of the impact zone, just as setting a maximum distance from the initial conflict (see Section 4.4). The number of new conflicts considered is directly related to the required compuation time.

\begin{tabular}{c|c|c}
\hline Strategy & $\begin{array}{c}\text { size of } \\
\text { DIZ }\end{array}$ & $\begin{array}{c}\text { Average size impact zone } \\
\text { (\# conflicts) }\end{array}$ \\
\hline Van Thielen et al. (2018) & unlimited & 357 \\
iCPS & unlimited & 292 \\
iCPS & $10 \mathrm{~km}$ & 113 \\
iCPS & $50 \mathrm{~km}$ & 261 \\
iCPS & $100 \mathrm{~km}$ & 288 \\
\hline
\end{tabular}

Table 5: The average number of conflicts in the dynamic impact zone, over all simulation runs. 


\subsection{Minimization of passenger delays}

Table 6 includes the passenger based extension as introduced in Section 4.6. The total passenger delay is the overall delay over all passengers in the study area, expressed in minutes. It can be decreased by $4.2 \%$ by considering 'minimizing passenger delays' as objective.

\begin{tabular}{c|c|c|c|c}
\hline $\begin{array}{c}\text { Objective } \\
\text { Function }\end{array}$ & $\begin{array}{c}\text { Train D } \\
\text { (in min) }\end{array}$ & $\begin{array}{c}\text { Pass D } \\
\text { (in min) }\end{array}$ & $\begin{array}{c}\text { Avg comp } \\
\text { time (in s) }\end{array}$ & $\begin{array}{c}\text { Max comp } \\
\text { time (in s) }\end{array}$ \\
\hline $\begin{array}{c}\text { Train delay (iCPS) } \\
\text { Passenger delay }\end{array}$ & $\begin{array}{c}359 \\
(+25.6 \%)\end{array}$ & $118071(-4.2 \%)$ & 2.2 & 34.1 \\
\hline
\end{tabular}

Table 6: Comparing passenger and train delays in the conflict prevention strategy.

We give some detail of the solution process and the consequence for the dispacthing actions by an example. Figure 8 shows time-distance plots of train 2206 . Every busy train is indicated by a blue tone, a quiet train by an orange tone and a non-passenger train by a green tone. In this run, there is a conflict between trains 2206 and 2078 in the station area of Denderleeuw. This conflict can be resolved in two ways: delaying train 2206 with 183 seconds or delaying 2078 with 196 seconds. The iCPS with objective minimizing train delays opts for delaying train 2206. When considering iCPS with objective minimizing the passenger delays, the option for delaying 2078 is preferred. This can also be clearly seen in Figure 8 where the pink block (2078) lies before the light blue (2206) in Figure 8a and after the light blue in Figure 8b. In the situation of Figure 8b compared to 8a, the passenger delay of train 2206 has decreased strongly from 108702 seconds to 19008 seconds over all passengers on the train, while the passenger delay of train 2078 has increased slightly from 11270 seconds to 14858 seconds over all passengers on this train. Figure 8 also shows that adjusting the objective function can lead to different rerouting choices, as trains 2056 and 2228 have changed routes from Figure 8a to Figure 8b. 


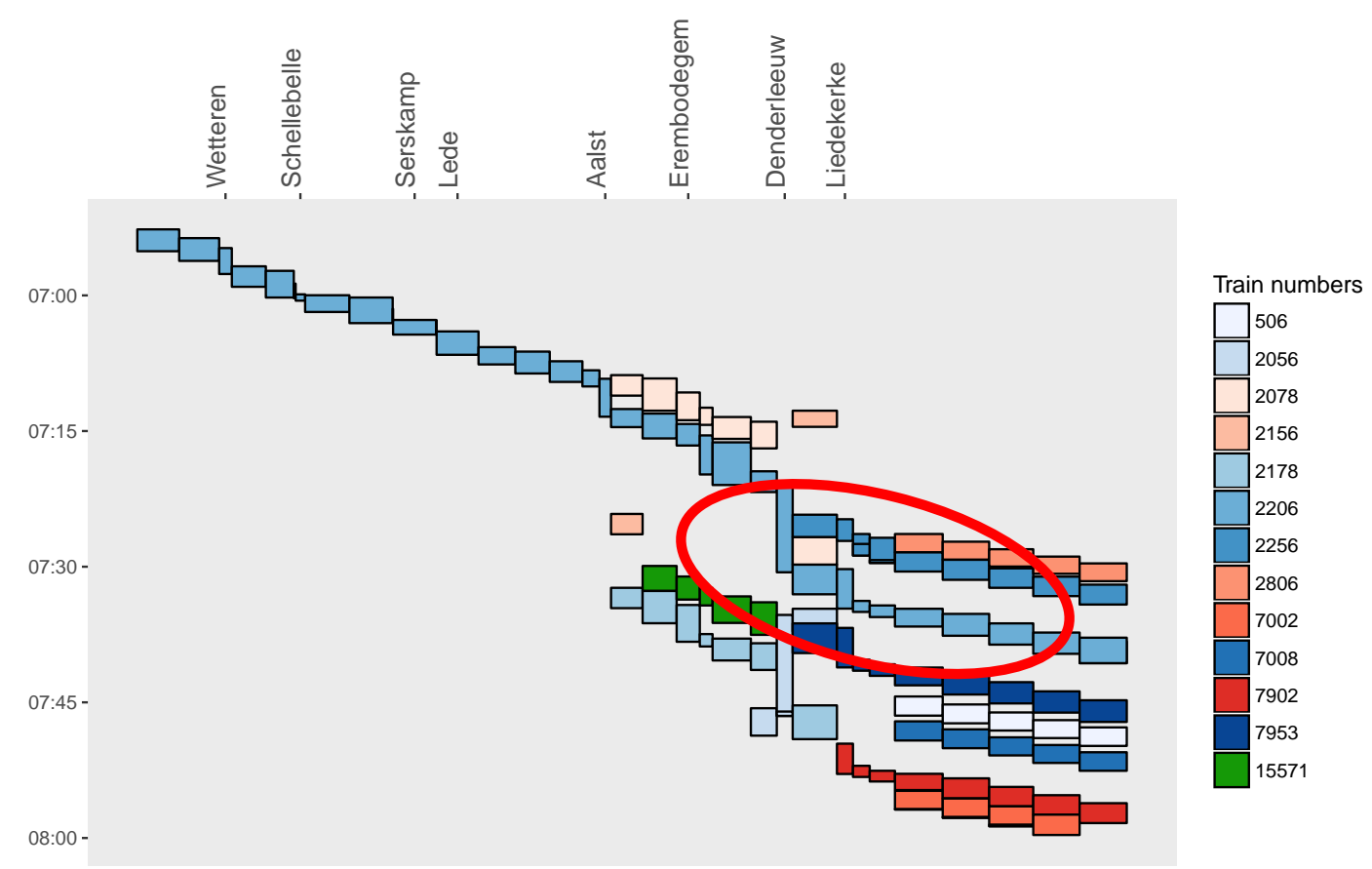

(a) Objective $=$ "minimize train delays"

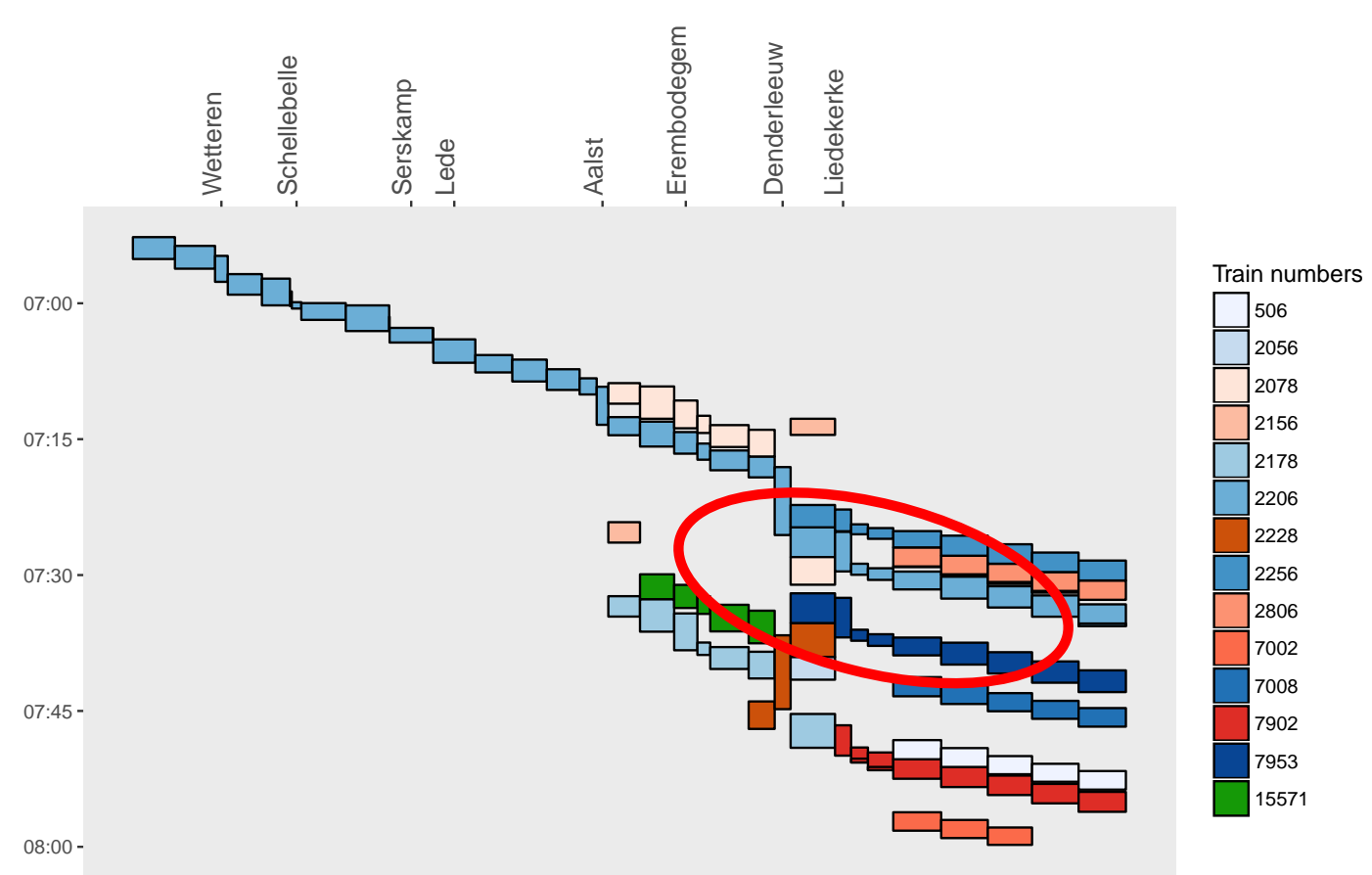

(b) Objective = "minimize passenger delays"

Figure 8: A time-distance plot showing the effects of changing the objective function on the busy train 2206 , indicated with a red ellipse. 


\subsection{Impact of train cancellations}

Table 7 shows the results of allowing trains to be canceled. For the 20 runs we consider, only in 9 of them one train is canceled, if the penalty for cancellations is 30 minutes (i.e. trains are canceled with a frequency of $9 / 20 / 240=0.018 \%$ ); only in 4 of the scenarios, in case the penalty for canceling is 60 minutes (i.e. trains are canceled with a frequency of 4 $/ 20 / 240=0.008 \%$ ). It can be seen from Table 7 that, although the cancellation penalty is considered during the progress examination, the train delays (including the penalties) increase when cancellations are allowed. This is obviously not a desired result, due to the short term effects of cancellations and the online setup of our study. A cancellation might decrease immediately some delays, but might cause some larger consequences further away.

During the progress examination of the decision to cancel a train, the delay caused by the canceled train (if not canceled) is systematically overestimated. As a result, the decision to cancel a train is sometimes taken too easily. Therefore, future research could focus more on adaptations of the DIZ heuristic for considering train cancellations.

\begin{tabular}{c|c|c|c|c|c}
\hline $\begin{array}{c}\text { Cancellation } \\
\text { penalty }\end{array}$ & $\begin{array}{c}\text { Train D without } \\
\text { penalty (in min) }\end{array}$ & $\begin{array}{c}\text { Train D with } \\
\text { penalty (in min) }\end{array}$ & $\begin{array}{c}\text { Pass D with } \\
\text { penalty (in min) }\end{array}$ & $\begin{array}{c}\text { Avg comp } \\
\text { time (in s) }\end{array}$ & $\begin{array}{c}\text { Max comp } \\
\text { time (in s) }\end{array}$ \\
\hline infinite & 359 & 359 & 123268 & 2.2 & 34.1 \\
30 min & 350 & 365 & 123680 & 3.6 & 34.8 \\
60 min & 355 & 371 & 123159 & 3.6 & 35.9 \\
\hline
\end{tabular}

Table 7: The effects of allowing trains to be canceled on the iCPS.

\subsection{Overall evaluation}

The percentage of times that the decision taken by a certain strategy is different from FCFS when there are several options possible, is included in Table 8. Rows report respectively on the benchmark from Van Thielen et al. (2018), the iCPS versions with unlimited and limited size of the dynamic impact zone; the iCPS taking care of passengers delays, and the two versions of the iCPS allowing cancellations if their delay is respectively 30 or 60 minutes. Especially when taking passengers into account, the FCFS option is less chosen. Another clear difference can be seen when the option of canceling a train is included. 


\begin{tabular}{c|c}
\hline Strategy & $\begin{array}{c}\text { Percentage not } \\
\text { FCFS option chosen }\end{array}$ \\
\hline Van Thielen et al. (2018) & $15.6 \%$ \\
iCPS-unlimited & $17.7 \%$ \\
iCPS-10km & $17.4 \%$ \\
iCPS-50km & $17.7 \%$ \\
iCPS-100km & $17.5 \%$ \\
iCPS-PASS & $23.8 \%$ \\
iCPS-CANC30 & $13.1 \%$ \\
iCPS-CANC60 & $12.8 \%$ \\
\hline
\end{tabular}

Table 8: The percentage of times FCFS is not chosen as an option in the conflict prevention strategy.

\subsection{Case Study 2: Interaction with different dispatching systems}

We also test the iCPS (with distance limit of $50 \mathrm{~km}$ ) on a larger microscopic network including the three provinces Antwerp, West Flanders and East Flanders in Belgium (see Figure 9). This area includes 191 station areas and 23917 block sections. Both freight and passenger trains are taken from the microscopic timetable of $17 / 03 / 2017$. During the time window between 7 and 8 a.m., there are at maximum 353 trains considered and 71 rolling stock connections are provided. In this case study, a part of the area is considered as a black box (see Section 4.5).

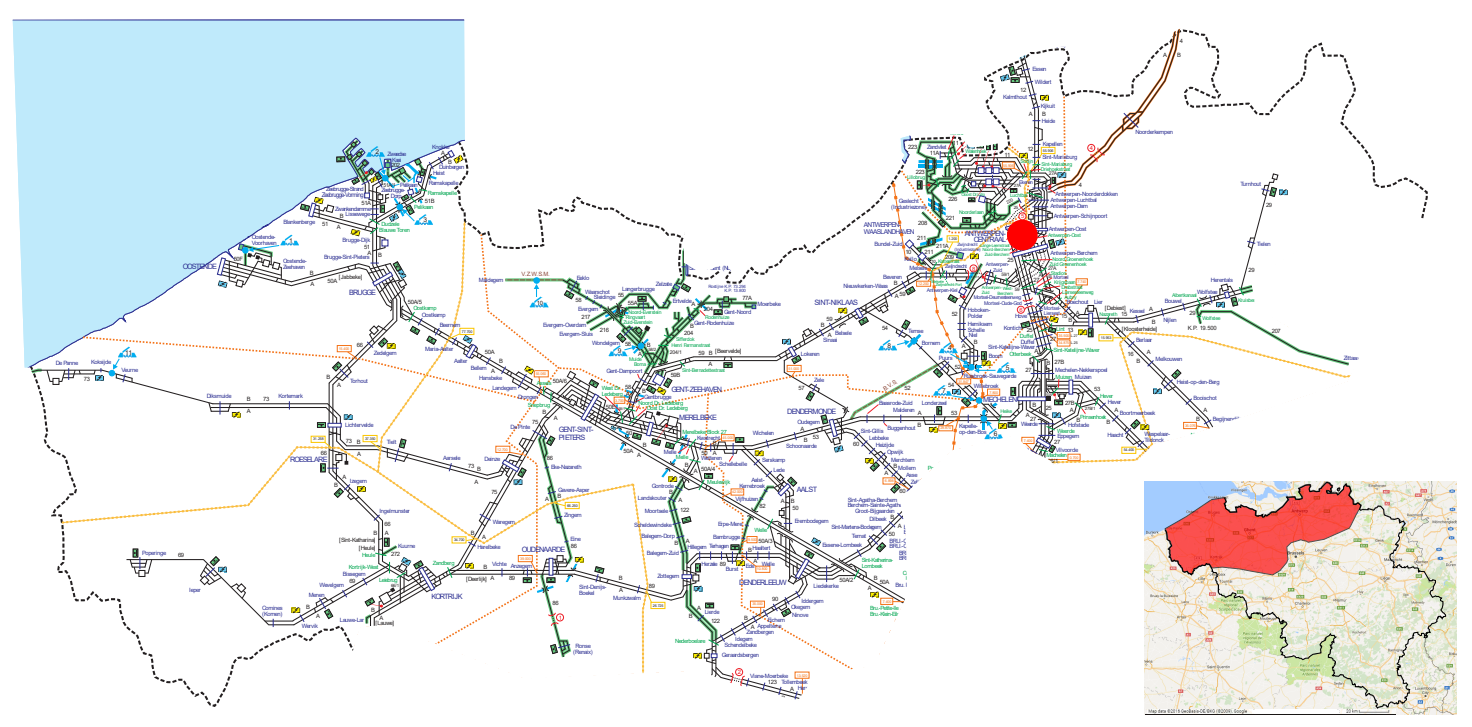

Figure 9: Study area consisting of the three provinces Antwerp, West and East Flanders. The red circle is the area considered as a black box (see Section 4.5) 
The results are shown in Table 9, where the basic dispatching strategies (FCFS and First-order) are compared to the extension of Van Thielen et al. (2018) to deal with rolling stock connections, and our iCPS method presented here (iCPS-50km). Clearly, both the extension of Van Thielen et al. (2018) to deal with rolling stock connections, and iCPS outperform the FCFS strategy on the larger network. The iCPS strategy performs approximately $45 \%$ better than the FCFS, and $11 \%$ better compared to the extension of Van Thielen et al. (2018), while requiring less computation time on average than this latter. As intended, iCPS performs better than our previous strategies and is adequate to be implemented in a real-world TMS, managing large and complex railway networks.

\begin{tabular}{c|c|c|c}
\hline Strategy & $\begin{array}{c}\text { Train D } \\
\text { (in min) }\end{array}$ & $\begin{array}{c}\text { Avg comp } \\
\text { time (in s) }\end{array}$ & $\begin{array}{c}\text { Max comp } \\
\text { time (in s) }\end{array}$ \\
\hline FCFS & 843 & $<0.1$ & $<0.1$ \\
First-order & $562(-33 \%)$ & 2.37 & 38.1 \\
Van Thielen et al. (2018) & $522(-38 \%)$ & 3.1 & 39.5 \\
iCPS-50km & $466(-45 \%)$ & 2.43 & 39.3 \\
\hline
\end{tabular}

Table 9: Testing the 'best' iCPS on the network of Antwerp, West and East Flanders.

\section{Conclusion}

This paper proposes an improved and extended Conflict Prevention Strategy (CPS) capable of resolving conflicts in real-time for large and complex networks. Our CPS consists of a rerouting optimization for station areas and a Dynamic Impact Zone (DIZ) heuristic that retimes/reorders trains. The CPS is adjusted to include rolling stock connections between trains to make the approach more realistic. The creation of the dynamic impact zone is improved such that it focuses even more on the relevant conflicts during the progress examination of the DIZ heuristic. Also, during the progress examination, conflicts are no longer resolved using FCFS, but by taking the best decision including the immediate impact in terms of delays. The DIZ heuristic is extended to include passenger numbers and to include the possibility of canceling a train. The objective function can either be minimizing train delays or passenger delays, depending on the necessary requirements. This could be considered as a rather straightforward extension, but we consider it as a crucial strength of our approach that the objective function can be modified easily.

This improved and extended CPS, iCPS, is tested on a large and complex network with a microscopic infrastructure and timetable from practice. Clearly, the improvements assure that the computation time remains limited and that the total train delay is further decreased with $6.3 \%$ on average. Compared to FCFS, this iCPS with rolling stock connections has on average $51 \%$ less train delays. When considering the total passenger delays, the iCPS can outperform FCFS by $22 \%$. Including the option of canceling a train is not often taken when the cancellation penalty is set to 30 minutes or to 60 minutes. 
The iCPS is also tested on an even larger network and compared to our previous CPS (see Van Thielen et al. (2018)), without any improvements or extensions considered, and to the first-order strategy and FCFS. Clearly, iCPS outperform the other strategies on this larger network by as much as $45 \%$. Moreover, the iCPS strategy improves the previous published CPS by $11 \%$ in train delays while requiring less computation time.

Any researcher wanting to monitor or evaluate efficiently the consequences of (the resolution of) a certain conflict, can benefit from our work. We indicate how to efficiently select the other conflicts (and trains) that should be considered and how these should be treated during the evaluation. Moreover, we show that it makes sense to use a distance limit when considering the consequences of a conflict.

The fact that our approach is a heuristic, brings obvious strengths and limitations. The most important limitation is that we cannot guarantee an optimal solution. However, we actually argue that an optimal solution can only be found in theory, when future progress of all trains is known deterministically. Moreover, our approach only makes a local evaluation of the consequences of a certain decision, not global evaluation. However, the dynamic impact zone tries to focus on the most relevant consequences. The strength is clearly the quality of the conflict resolution and that the size of the dynamic impact zone, and thus the required computation time, can be controlled easily.

In future research, a heuristic method for the rerouting optimization can be included to lower the computation time in station areas. The computation time can also be reduced by applying a parallel algorithm on the DIZ heuristic, where the progress examination of the possible resolutions can be computed in parallel. To further close the gap between scientific research and practice, more research on real-time railway management should focus on techniques (and computation times) that can actually be implemented in practice, also on large scale networks.

\section{References}

Albrecht, A.R., Panton, D.M. and Lee, D.H., Rescheduling rail networks with maintenance disruptions using problem space search. Computers \& Operations Research, 40, 387-398 (2013)

Borndörfer, R., Klug, T., Lamorgese, L., Mannino, C., Reuther, M. and Schlechte, T, Recent Succes Stories on integrated optimization of railway systems. Transportation Research Part C, 74, 196-211 (2017)

Bettinelli, A., Santini, A., and Vigo, D. (2017). A real-time conflict solution algorithm for the train rescheduling problem. Transportation Research Part B: Methodological,106:237-265.

Cacchiani, V., Huisman, D., Kidd, M., Kroon, L., Toth, P., Veelenturf, L. and Wagenaar, $\mathrm{J}$, An overview of recovery models and algorithms for real-time railway rescheduling. Transportation Research Part B, 63, 15-37 (2014) 
Caimi, G., Fuchsberger, M., Laumanns, M. and Lüthi, M, A model predictive control approach for discrete-time rescheduling in complex central railway station areas. Computers \& Operations Research, 39, 2578-2593 (2012)

Corman, F., D'Ariano, A., Pacciarelli, D. and Pranzo, M., Centralized versus distributed systems to reschedule trains in two dispatching areas. Public Transport, 2 (3), 219-247 (2010b)

Corman, F., D'Ariano, A., Pacciarelli, D. and Pranzo, M., Optimal inter-area coordination of train rescheduling decisions. Transportation Research Part E, 48, 71-88 (2012)

Corman, F. and D'Ariano, A., Assessment of Advanced Dispatching Measures for Recovering Disrupted Railway Traffic Situations. Transportation Research Record: Journal of the Transportation Research Board, 2289, 1-9 (2012)

Corman, F. and Meng, L. A review of online dynamic models and algorithms for railway traffic control. IEEE International Conference on Intelligent Rail Transportation (ICIRT), 2013, Beijing, 128-133 (2013)

Corman, F. and Quaglietta, E., Closing the loop in railway traffic control: framework design and impacts on operations. Transportation Research Part C, 54, 15-39 (2015)

Corman, F., D'Ariano, A., Marra, A., Pacciarelli, D. and Samà, M, Integrating train scheduling and delay management in real-time railway traffic management. Transportation Research Part E, 105, 213-239 (2017)

D'Ariano, A., Pranzo, M. and Hansen, I.A., Conflict resolution and train speed coordination for solving real-time timetable perturbations. IEEE Transactions on Intelligent Transportation Systems, 8(2), 208-222 (2007)

Dolder, U., Krista, M. and Voelcker, M. RCS - rail control system, realtime train run simulation and conflict detection on a netwide scale based on updated train positions. In Proceedings of the 3rd International Seminar on Railway Operations Modelling and Analysis, RailZurich (2009)

Hansen, I. and Pachl, J, Railway timetable and traffic: analysis, modelling, simulation. Eurail Press (2008)

Fischetti, M, Monaci, M (2017) Using a general-purpose Mixed-Integer Linear Programming solver for the practical solution of real-time train rescheduling, European Journal of Operational Research, Volume 263, Issue 1,2017,Pages 258-264,

Lamorgese, L. and Mannino, C., An exact decomposition approach for the real-time train dispatching problem. Operations Research, 63 (1), 48-64 (2015)

Lamorgese, L., Mannino, C. and Piacentini, M., Optimal train dispatching by Benders'-like reformulation. Transportation Science, 50(3), 910-925 (2016) 
Lamorgese L, Mannino C, Pacciarelli P , Törnquist Krasemann J (2018) Train Dispatching in R. Borndörfer et al. (eds.), Handbook of Optimization in the Railway Industry, International Series in Operations Research and Management Science 268.

Luan, X., Meng, L., Corman F., Non-discriminatory train dispatching in a rail transport market with multiple competing and collaborative train operating companies, Transportation Research, Part C (80) 148-174 (2017)

Louwerse, I. and Huisman, D., Adjusting a railway timetable in case of partial or complete blockades. European Journal of Operational Research, 235(3), 583-593 (2014)

Josyula, S., Törnquist Krasemann, J., Lundberg, L., (2018). A Parallel Algorithm for Train Rescheduling, Transportation Research Part C Vol. 95, pp. 545-569.

Mascis, A. and Pacciarelli, D., Job-shop scheduling with blocking and no-wait constraints. European Journal of Operations Research, 143, 598-517 (2002)

Meng, L. and Zhou, X., Robust single-track dispatching model under a dynamic and stochastic environment: a scenario-based rolling horizon solution approach. Transportation Research Part B, 45, 1080-1102 (2011)

Montigel M (2009) Operations control system in the Lötschberg base tunnel. Eur Rail Technol Rev 49(2):42- 44. ISSN: 0079-9548

Pellegrini, P., Marlière, G. and Rodriguez, J., A detailed analysis of the actual impact of real-time railway traffic management optimization. Journal of Rail Transport Planning \& Management, 6, 13-31 (2016)

Quaglietta, E., Corman, F. and Goverde, R.M.P., Stability analysis of railway dispatching plans in a stochastic and dynamic environment. Journal of Rail Transport Planning \& Management, 3, 137-149 (2013)

Quaglietta, E., Pellegrini, P., Goverde, R.M.P., Albrecht, T., Jaekel, B., Marlière, G., Rodriguez, J., Dollevoet, T., Ambrogio, B., Carcasole, D., Giaroli, M. and Nicholson, G., The ON-TIME real-time railway traffic management framework: A proof-of-concept using a scalable standardized data communication architecture. Transportation Research Part C, 63, 23-50 (2016)

Samà, M., Meloni, C., D'Ariano, A. and Corman, F., A multi-criteria decision support methodology for real-time train scheduling. Journal of Rail Transport Planning \& Management, 5(3), 146-162 (2015)

Samà, M., D'Ariano, A., Corman, F. and Pacciarelli, D., A variable neighbourhood search for fast train scheduling and routing during disturbed railway traffic situations. Computers \& Operations Research, 78, 480-499 (2017) 
Törnquist, J. and Persson, J.A., N-tracked railway traffic re-scheduling during disturbances. Transportation Research Part B, 41, 342-362 (2007)

Van Thielen, S., Corman, F. and Vansteenwegen, P., Considering a dynamic impact zone for real-time railway traffic management. Transportation Research Part B, 111, 39-59 (2018)

Van Thielen, S., 2019. "Conflict prevention strategies for real-time railway traffic management", PhD Thesis. KU Leuven. 